PROCEEDINGS OF THE

AMERICAN MATHEMATICAL SOCIETY

Volume 137, Number 7, July 2009, Pages 2329-2333

S 0002-9939(08)09788-8

Article electronically published on December 22, 2008

\title{
MEROMORPHIC FUNCTIONS WITH LINEARLY DISTRIBUTED VALUES AND JULIA SETS OF RATIONAL FUNCTIONS
}

\author{
WALTER BERGWEILER AND ALEXANDRE EREMENKO
}

(Communicated by Mario Bonk)

\begin{abstract}
If the preimage of a four-point set under a meromorphic function belongs to the real line, then the image of the real line is contained in a circle in the Riemann sphere. We include an application of this result to holomorphic dynamics: if the Julia set of a rational function is contained in a smooth curve, then it is contained in a circle.
\end{abstract}

If the full preimage of a two-point set under a rational function belongs to the real line, then the function maps the real line to a circle (on the Riemann sphere). We prove an analog of this simple fact for transcendental functions meromorphic in the plane.

Theorem 1. Let $f$ be a meromorphic function such that the preimage of three points belongs to the real line. Then $f$ maps the real line into a circle, unless

$$
f(z)=L\left(\frac{1-e^{i\left(c_{1} z-b_{1}\right)}}{1-e^{i\left(c_{2} z-b_{2}\right)}}\right),
$$

where $L$ is a fractional-linear transformation, and $c_{j}, b_{j}$ are real numbers.

Corollary. If the preimage of four points belongs to the real line, then $f$ maps the real line into a circle.

The circle of the corollary does not have to pass through the four points, as the example $f(z)=e^{i z}$ shows: $f$ maps the real line into the unit circle, and the preimage of $\left\{0, e^{i \alpha}, e^{i \beta}, \infty\right\}, \alpha, \beta \in \mathbf{R}$, is on the real line, but in general there is no circle passing through these four points.

We give an application of Theorem 1 to holomorphic dynamics. Fatou [3, no. 56, p. 250] proved the following:

Let $f$ be a rational function, and suppose that some relatively open subset of the Julia set $J(f)$ is a simple smooth curve. Then the Julia set is either a circle or an arc of a circle.

Here we call a curve smooth if it has a tangent at every point.

Received by the editors September 2, 2008.

2000 Mathematics Subject Classification. Primary 30D35.

The first author was supported by the G.I.F., the German-Israeli Foundation for Scientific Research and Development, Grant G-809-234.6/2003; the EU Research Training Network CODY; and the ESF Research Networking Programme HCAA.

The second author was supported by NSF grant DMS-0555279.

(C)2008 American Mathematical Society Reverts to public domain 28 years from publication 
The assumption of Fatou's theorem has been substantially relaxed by Hamilton [5]: if a relatively open subset of the Julia set is a curve of Hausdorff dimension 1 , then the Julia set is a circle or an arc of a circle.

We generalize this result of Fatou in another direction:

Theorem 2. If a relatively open subset of the Julia set of a rational function is contained in a smooth curve, then the Julia set is contained in a circle.

This theorem was first proved in 2 using a different method. Our paper was inspired by conversations on Theorem 2 with Sebastian van Strien, whom we thank.

We remark that we cannot replace "smooth curve" by "curve of Hausdorff dimension 1" in Theorem 2. In fact, Hamilton [6] constructed a rational function whose Julia set is a Cantor subset of a rectifiable curve, but does not belong to any circle.

In the discussion of examples of Julia sets we assume that the circle in question is the real line. Our first example is a "Blaschke product" (a rational function $f$ such that both upper and lower half-planes are invariant under the second iterate $f^{2}$ ). The Julia set of such a function can be either a Cantor set on the real line or coincide with the real line.

The second class of examples consists of certain real polynomials. Suppose that the Julia set of a polynomial $f$ of degree $d$ is contained in the real line, and let $[a, b]$ be the convex hull of the Julia set. Then $f$ is a real function, $f(\{a, b\}) \subset\{a, b\}$, all finite critical points of $f$ belong to $[a, b]$, and all critical values belong to the complement of $(a, b)$. It is easy to show that these properties are also sufficient for the Julia set to lie on the interval $[a, b]$ of the real line. If all critical values belong to the set $\{a, b\}$, then $f$ is conjugate to a Chebyshev polynomial or to the negative of a Chebyshev polynomial.

There are also examples which are neither Blaschke products nor polynomials; the simplest of them is $f(z)=\left(z^{2}-c\right) /(1+\varepsilon z)$, where $c<-2$ and $\varepsilon$ is real and small enough. It is desirable to give some classification of rational functions whose Julia sets are contained in the real line.

For the proof of Theorem 1 we need the following result.

Lemma. Let $f$ be a meromorphic function and let $a_{1}, a_{2}, a_{3}$ be three points in the Riemann sphere such that all solutions of the equations

$$
f(z)=a_{j}, \quad j=1,2,3
$$

are real. Then either $f$ maps the real line into the circle passing through the $a_{j}$ or the order of $f$ is at most 1 .

Proof. We use standard facts and the notation of Nevanlinna theory and Tsuji characteristics for a half-plane [4. One could give a more elementary but longer proof by following the method of Edrei's paper [1].

Without loss of generality we assume that $\left(a_{1}, a_{2}, a_{3}\right)=(0,1,-1)$. We have to prove that $f$ is either real or has order at most 1 . Let $g(z)=\overline{f(\bar{z})}$. As all $a_{j}$-points of $f$ are real, we conclude that $f$ and $g$ share $a_{j}$-points, counting multiplicity. If $f=g$, then $f$ is real. If $f \neq g$, then we have

$$
N(r, 0, f-g) \geq N\left(r, a_{1}, f\right)+N\left(r, a_{2}, f\right)+N\left(r, a_{3}, f\right) .
$$

On the other hand,

$$
N(r, 0, f-g) \leq T(r, f-g)+O(1) \leq 2 T(r, f)+O(1) .
$$


Combining these two inequalities and using the First Main Theorem of Nevanlinna, we conclude that

$$
T(r, f) \leq m\left(r, a_{1}, f\right)+m\left(r, a_{2}, f\right)+m\left(r, a_{3}, f\right)+O(1) .
$$

As $f$ omits three values in the upper and lower half-planes, we conclude that its Tsuji characteristics in both half-planes satisfy

$$
\mathfrak{m}\left(r, a_{j}, f\right)=\mathfrak{T}(r, f)+O(1)=O(\log r) ;
$$

see [4, Ch. III, §3]. Now we use the estimate of the Nevanlinna proximity functions in terms of the Tsuji proximity functions 4, Ch. VI, Lemma 5.2]

$$
\int_{R}^{\infty} \frac{m(r, a, f)}{r^{3}} d r \leq \int_{R}^{\infty} \frac{\mathfrak{m}^{*}(r, a, f)}{r^{2}} d r, \quad R>0,
$$

where $\mathfrak{m}^{*}$ is the sum of the Tsuji proximity functions of the upper and lower halfplanes. Using (3) and (2), we conclude that

$$
\int_{R}^{\infty} \frac{T(r, f)}{r^{3}} d r=O\left(R^{-1} \log R\right),
$$

and as $T$ is increasing, we obtain $T(R, f)=O(R \log R)$; that is, the order of $f$ is at most 1 .

Proof of Theorem 1. Without loss of generality we assume that the three points are $0,1, \infty$. Then we have to prove that our function $f$ is real, unless it is of the form (1).

Put $g(z)=\overline{f(\bar{z})}$. Then $f$ and $g$ share three values: $0,1, \infty$ (counting multiplicity). We conclude that

where $u$ and $v$ are some entire functions.

$$
\frac{f}{g}=e^{u} \quad \text { and } \quad \frac{f-1}{g-1}=e^{v}
$$

By solving these equations with respect to $f$ and $g$ we obtain

$$
g=\frac{1-e^{v}}{e^{u}-e^{v}} \quad \text { and } \quad f=\frac{1-e^{v}}{1-e^{v-u}} .
$$

Our lemma implies that if $f$ is not real, then $f$ is of order at most 1 , so both functions $u$ and $v$ are affine in this case. It is easy to see now that any nonconstant function $u$ or $v$ has to be of the form $i(c z+b)$, where the coefficients $c$ and $b$ are real.

If both $u$ and $v$ are nonconstant or both of them are constant, then $f$ is of the form (1).

If one of the functions $u, v$ is constant and the other is not, then $f(z)=$ $L\left(e^{i(c z+b)}\right)$, where $L$ is a fractional-linear transformation and $c \in \mathbf{R} \backslash\{0\}$, so $f$ maps the real line into a circle.

Proof of the Corollary. It is enough to consider exceptional functions of the form

$$
f(z)=\frac{1-e^{i z}}{1-e^{i(c z+b)}}
$$

where $c \notin\{0,1\}$. (If $c \in\{0,1\}$, then $f(z)=L\left(e^{i z}\right)$, where $L$ is a fractional-linear transformation, and $f$ maps the real line into a circle.) Suppose that the preimage of some $a \in \mathbf{C} \backslash\{0,1, \infty\}$ belongs to the real line. We are going to prove that $f$ maps the real line into a circle. 
So we assume that the equation

$$
1-a-e^{i z}+a e^{i(c z+b)}=0
$$

has only real zeros. The left-hand side of this equation is an entire function of order 1 with only real zeros, so by Hadamard's factorization theorem, it is a product of a real entire function $w$ and $e^{-i \gamma z}$ with some real $\gamma$. Thus the ratio of the left-hand side of (4) and $e^{-i \gamma z}$ is a real entire function $w$ that is $\overline{w(\bar{z})}=w(z)$, which gives

$$
\begin{aligned}
w(z) & \equiv(1-a) e^{i \gamma z}-e^{i(1+\gamma) z}+a e^{i b} e^{i(c+\gamma) z} \\
& \equiv(1-\bar{a}) e^{-i \gamma z}-e^{-i(1+\gamma) z}+\bar{a} e^{-i b} e^{-i(c+\gamma) z}
\end{aligned}
$$

Such an identity can only hold for trivial reasons, because any set of functions $e^{i \alpha z}$ is linearly independent. In particular, we conclude that

$$
\gamma \in\{0,-1-\gamma,-c-\gamma\}
$$

We examine all three possibilities.

1. $\gamma=0$ implies $a=\bar{a}$. Then $c=-1$ and $a e^{i b}=-1$. As we assume that $a \neq 1$ and $b$ is real, the only possibility is that $a=-1$ and $e^{i b}=1$. Then $f(z)=$ $\left(1-e^{i z}\right) /\left(1-e^{-i z}\right)=-e^{i z}$ maps the real line into the unit circle.

2. $\gamma=-1-\gamma$ implies $\gamma=-1 / 2$ and then $c \in\{0,1\}$, the cases we excluded before.

3. $\gamma=-c-\gamma$ implies $\gamma=-c / 2$ and then again $c \in\{0,1\}$, the cases excluded before.

Proof of Theorem 2. Let $V$ be an open set such that the intersection $J \cap V$ is nonempty and is contained in a smooth simple curve $\gamma$. Let $p \in J \cap V$ be a repelling periodic point. Replacing $f$ by some iterate we may assume without loss of generality that $p$ is fixed by $f$.

Consider the Poincaré function $F$ associated with $f$ and $p$. This function satisfies

$$
F(\lambda z)=f(F(z)), \quad F(0)=p, \quad F^{\prime}(0)=1
$$

and is meromorphic in C. Here $\lambda=f^{\prime}(p),|\lambda|>1$.

Let $I=F^{-1}(J)$ be the preimage of the Julia set under $F$. Then $\lambda I=I$. As $F$ is conformal at 0 , there is a $\lambda^{-1}$-invariant neighborhood $U$ of 0 such that $I \cap U$ belongs to a smooth simple curve $\Gamma$ passing through 0 . This curve is defined by the property that $F(\Gamma) \subset \gamma$. It is easy to see that whenever a subset of a smooth curve is invariant with respect to multiplication by $\lambda^{-1}$, this subset has to belong to a straight line through 0 and $\lambda$ has to be real. (This argument was used by Fatou 3 , no. 46, p. 229].) Thus the intersection of $I$ with a neighborhood of 0 belongs to a straight line. Then the $\lambda$-invariance of $I$ implies that the whole set $I$ is contained in a straight line. As the $F$-preimages of all points of the Julia set belong to $I$, an application of the corollary to Theorem 1 shows that the Julia set is contained in a circle.

\section{ACKNOWLEDGEMENT}

We thank the referee for drawing our attention to [5]. 


\section{REFERENCES}

1. A. Edrei, Meromorphic functions with three radially distributed values, Trans. Amer. Math. Soc., 78 (1955) 276-293. MR0067982 (16:808d)

2. A. Eremenko and S. van Strien, Rational functions with real multipliers, preprint, arXiv:0810.2260.

3. P. Fatou, Sur les équations fonctionnelles (Troisième Mémoire), Bull. Soc. Math. France, 48 (1920) 208-314. MR:1504797

4. A. Goldberg and I. Ostrovskii, Distribution of values of meromorphic functions (Russian). Nauka, Moscow, 1970. English translation: Amer. Math. Soc., Providence, RI, 2008. MR0280720 (43:6439)

5. D. Hamilton, Length of Julia curves, Pacific J. Math., 169 (1995) 75-93. MR1346247 (96m:30038)

6. D. Hamilton, Rectifiable Julia curves, J. London Math. Soc., 54 (1996) 530-540. MR1413896 (98j:30022)

Mathematisches Seminar, Christian-Albrechts-Universität ZU Kiel, Ludewig-MeynStr. 4, D-24098 Kiel, Germany

E-mail address: bergweiler@math.uni-kiel.de

Department of Mathematics, Purdue University, West Lafayette, Indiana 47907

E-mail address: eremenko@math.purdue.edu 